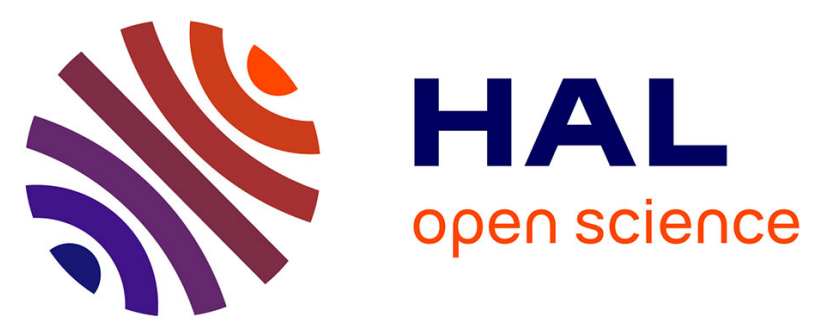

\title{
A Moment-based Distributionally Robust Optimization Model for Air Traffic Flow Management
} Bin Hao, Kaiquan Cai, Yiping Fang, Abdelghani Fadil, Daozhong Feng

\section{To cite this version:}

Bin Hao, Kaiquan Cai, Yiping Fang, Abdelghani Fadil, Daozhong Feng. A Moment-based Distributionally Robust Optimization Model for Air Traffic Flow Management. 2021 IEEE/AIAA 40th Digital Avionics Systems Conference (DASC), Oct 2021, San Antonio, United States. pp.1-7, 10.1109/DASC52595.2021.9594502 . hal-03441646

\section{HAL Id: hal-03441646 https://hal.science/hal-03441646}

Submitted on 3 Dec 2021

HAL is a multi-disciplinary open access archive for the deposit and dissemination of scientific research documents, whether they are published or not. The documents may come from teaching and research institutions in France or abroad, or from public or private research centers.
L'archive ouverte pluridisciplinaire HAL, est destinée au dépôt et à la diffusion de documents scientifiques de niveau recherche, publiés ou non, émanant des établissements d'enseignement et de recherche français ou étrangers, des laboratoires publics ou privés. 


\title{
A Moment-based Distributionally Robust Optimization Model for Air Traffic Flow Management
}

\author{
$1^{\text {st }}$ Bin Hao \\ School of Electronic and Information \\ Engineering \\ Beihang University \\ Beijing, China \\ haobinn@buaa.edu.cn \\ $4^{\text {th }}$ Abdelghani Fadil \\ School of Electronic and Information \\ Engineering \\ Beihang University \\ Beijing, China \\ fadilabdelghani@buaa.edu.cn
}

\author{
$2^{\text {nd }}$ Kaiquan Cai \\ School of Electronic and Information \\ Engineering \\ Beihang University \\ Beijing, China \\ caikq@buaa.edu.cn
}

\author{
$3^{\text {rd }}$ Yi-Ping Fang \\ Laboratoire Génie Industriel \\ Université Paris-Saclay \\ Paris, France \\ yiping.fang@centralesupelec.fr
}

\author{
$5^{\text {th }}$ Daozhong Feng \\ School of Electronic and Information \\ Engineering \\ Beihang University \\ Beijing, China \\ daozhongfeng@buaa.edu.cn
}

\begin{abstract}
The Air Traffic Flow Management (ATFM) is considered as an effective method for air safety and efficiency guarantee. However, as one of the vital part of flight efficiency, fuel consumption is easily affected by external factors induced by various uncertainties such as severe weather conditions, which impact decision making process. Therefore, a distributionally robust mixed integer programming model for ATFM problem (DR-ATFM) is introduced in this paper to handle the uncertainty of fuel consumption while minimizing the total cost of fuel consumption, flight cancellation and flight delays. By exploiting the moment information of the fuel consumption data, a moment ambiguity set is constructed which characterizes the actual distribution of fuel consumption influctation. Based on this, an equivalent reformulation of DR-ATFM is derived to transform the problem into a mathematically solvable one, which is further solved through a cutting plane-based decomposition algorithm proposed in this paper. Finally, the effectiveness and robustness of the method are verified based on computational results of small-sized instances.
\end{abstract}

Index Terms-ATFM, uncertainty, distributionally robust optimization, cutting plane-based decomposition

\section{NOMENCLATURE}

\section{A. Sets}

$A$

$A_{f}$

$A_{\text {out }}^{f}(s)$

$A_{i n}^{f}(s)$

$D$

F

$H$

$P$

$P^{f}$
Set of all airways

Set of airways for flight $f$

Set of airways outbound from waypoint $s$

Set of airways inbound to waypoint $s$

Set of candidate distributions for fuel consumption

Set of flights in network

Set of height levels for airways

Set of waypoints and airports

Set of waypoints and airports for flight $f$
$T$

$\operatorname{dep}_{f}$

Set of time periods

Set of departure airways for flight $f$

B. Parameters

$D_{f} \quad$ Cancellation cost for flight $f$

$T \_\min _{a}^{f, h} \quad$ The minimal traversing time of airway $a$ at height $h$ for flight $f$

$\operatorname{arr}_{f} \quad$ Arrival airway for flight $f$

orign $_{f} \quad$ Departure airport for flight $f$

dest $_{f} \quad$ Arrival airport for flight $f$

$g_{t}^{f} \quad$ Unit time cost of delay for flight $f$

$\Delta_{h} \quad$ The maximal height difference between airways

C. Variables

$e_{a, t}^{f, h}$

Binary decision variable to indicate if flight $f$ enters airway $a$ at height $h$ in period $t$. "1" if it enters and "0" otherwise

$\tau, \alpha, \beta \quad$ Dual variables of DR-ATFM model

\section{Random Parameters}

$\xi_{a, t}^{h} \quad$ Continuous variables indicating the fuel consumption of airway $a$ in period $t$ at height $h$

\section{INTRODUCTION}

Air traffic demand is growing rapidly while airspace capacity improvement is stagnating, leading to increased demandcapacity imbalances and frequent flight delays. In 2019, the average delay per flight was 13.1 minutes in Europe, 12.9 minutes in the United States and about 14 minutes in China 
[1]-[3]. These delays have led to undesirable consequences such as unpleasant passenger experiences and economic losses of airlines. Air Traffic Flow Management (ATFM) is considered as an effective way to alleviate flight delays by means of matching air traffic flow to sector capacity. In ATFM field, a large amount of studies have been done based on airports network, airspace sectors network [4] (nodes include airports and sectors), or air routes network [5]. As for ATFM problem, air routes network is the most common form in which civil flight activities are abstracted and modeled. However, in the operation level, air traffic flow contains inherent uncertainty brought by numerous factors including maneuvering, weather and ATC controllers' decision [6]. Thus, how to quantify the impact of uncertainty in air routes network is a major difficulty scholars face in the field.

Previous works for solving the above challenge could be roughly divided into two categories: stochastic programming (SP) and robust optimization (RO). SP utilizes assumed probability distributions to describe different kinds of uncertain scenarios and solves ATFM problem. Alonso-Ayuso et al. [7] proposed a ATFM model taking into account of uncertainty of airport arrival and departure capacity in several assumed scenarios. With system capacity being modeled stochastically, Marron [8] formulated a model to plan rerouting strategies for aircrafts to alleviate en-route congestion while using DantzigWolfe decomposition to reduce computational complexity.

Though SP approach is simple and effective for ATFM problems [9], [10], it can be hard to implement, if not impossible, when decisions need to be made before the full information related to flights is awared. In contrast, RO requires less information and could offer a more robust way to capture the uncertain information than SP in min-max cost approach. Saraf et al. [11] presented a Monte Carlo simulation-based approach for quantifying the uncertainties of demand and capacity, and assessed reasonable solutions robust to above two factors. Bertsimas et al. [12] proposed a robust integer programming model that allows controlling the degree of conservatism. When the cost coefficients and the data are not all known. However, RO typically produces over-conservative solutions for the ATFM problem in the worst-case scenarios, which results in inefficient use of airspace and waste of resources.

To effectively address the over-conservative problem, a moment-based distributionally robust optimization (DRO) approach is utilized in this paper for ATFM problem with uncertainty (DR-ATFM). DRO approaches have been applied to solve other problems such as robust optimal network design [14] and optimal power system operations [15], [16]. DRO constructs a set of distributions containing a serial of uncertain parameters by analyzing data based on which optimal decisions could effectively avoid over-conservatism [13]. The contributions of this paper include:

- Considering the uncertainty of fuel consumption in air routes, a moment-based distributionally robust mixed integer programming (DRMIP) model for the ATFM problem is developed. Within the problem, the total cost of fuel consumption, flight cancellation and flight delays are minimized. And comprehensive traffic management measures are considered such as ground holding, airborne holding and reroute action.

- The DRMIP model is reformulated to a mathematical uniform one and then solved by a cutting plane-based decomposition algorithm.

- With out-of-sample simulation data, the effectiveness and robustness of the proposed model are verified through small-sized instances.

The paper is organized as follows. In Section II, the DRATFM model and the ambiguity set of fuel consumption probability distributions are presented. The costs of arrival delay, fuel consumption and flight cancellation are minimized in the model. In Section III, the DR-ATFM model is reformulated and a cutting plane-based decomposition algorithm is derived. In Section IV, the effectiveness and robustness of the method are verified by computational results of small-sized instances. Section V summarizes the paper and discusses future work.

\section{MAThematical Formulation}

In this section, the DR-ATFM problem is introduced in detail. The model minimizes the costs of arrival delay, fuel consumption and flight cancellation while ensuring that flights' constraints are satisfied. An air routes network in which flights can re-route by selecting airways is given. It is considered that the fuel consumption of flying through an airway is uncertain, i.e., only the related information from limited data could be obtained. Then, a compact form of the problem is presented for the sake of algorithm deduction. And the ambiguity set of fuel consumption is constructed.

Before introducing the mathematical model of DR-ATFM problem, two concepts should be clarified here: (1) air routes network: each waypoint or airport can be represented as a node in the network. If a flight is planned to fly from one node to another, there will be a directed arc between these two nodes; (2) fuel consumption: fuel consumption is related to many parameters such as flight type, travel time and others, which makes it sensitive and thus typical as a way of evaluating the trajectory. For each airway, the fuel consumption is assumed identical for all flights and to be a random variable due to uncertain factors such as convective weather.

\section{A. DR-ATFM Mathematical Model}

The DR-ATFM model is presented as follows.

$$
\begin{gathered}
\min _{e_{a, t}^{f, h}} \max _{P \in D} E_{P}\left[\sum_{f \in F}\left(c_{f}+g_{f}+d_{f}\right)\right] \\
\text { s.t. } \quad \sum_{t \in T} e_{a r r_{f}, t}^{f, h_{0}}=\sum_{t \in T} e_{a, t}^{f, h_{0}} \leq 1 \quad \forall f \in F, a \in d e p_{f} \\
\sum_{f \in F} e_{a, t}^{f, h} \leq 1 \quad \forall a \in A, t \in T, h \in H
\end{gathered}
$$




$$
\begin{aligned}
& \sum_{b \in A_{\text {out }}^{f}(s)} \sum_{h \in H} \sum_{t \in T} e_{b, t}^{f, h}=\sum_{a \in A_{i n}^{f}(s)} \sum_{h \in H} \sum_{t \in T} e_{a, t}^{f, h} \\
& \forall f \in F, s \in\left\{P^{f} \backslash \operatorname{orign}^{f} \backslash \operatorname{dest}^{f}\right\} \\
& \sum_{a \in A_{\text {out }}^{f}(s)} \sum_{h \in H} \sum_{t \in T} e_{a, t}^{f, h} \leq 1 \quad \forall f \in F, s \in P \\
& \sum_{a \in A_{\text {out }}^{f}(s)} \sum_{h \in H} \sum_{t \in T} t \cdot e_{a, t}^{f, h}-\sum_{b \in A_{\text {in }}^{f}(s)} \sum_{h \in H} \sum_{t \in T} t \cdot e_{b, t}^{f, h} \\
& \geq \sum_{b \in A_{i n}^{f}(s)} \sum_{h \in H} \sum_{t \in T} T_{-} \min _{b}^{f, h} \cdot e_{b, t}^{f, h} \\
& \forall s \in\left\{P^{f} \backslash \operatorname{orign}^{f}\right\}, f \in F
\end{aligned}
$$

$$
\begin{gathered}
\left|\sum_{a \in A_{\text {in }}^{f}(s)} \sum_{h \in H} \sum_{t \in T} h \cdot e_{a, t}^{f, h}-\sum_{b \in A_{\text {out }}^{f}(s)} \sum_{h \in H} \sum_{t \in T} h \cdot e_{b, t}^{f, h}\right| \leq \Delta_{h} \\
\forall f \in F, s \in\left\{P^{f} \backslash \text { orign }{ }^{f} \backslash \text { dest }^{f}\right\}
\end{gathered}
$$

$$
\begin{gathered}
\sum_{h \in H} \sum_{t \in T} e_{d e p_{f}, t}^{f, h}=\sum_{h \in H} \sum_{t \in T} e_{a r r_{f}, t}^{f, h}=0 \quad \forall f \in F \\
e_{a, t}^{f, h} \in\{0,1\} \quad \forall f \in F, t \in T, h \in H, a \in A_{f}
\end{gathered}
$$

where $c_{f}, g_{f}, d_{f}$ represent the costs of fuel consumption, arrival delay and cancellation, respectively, for flight $f$. They are described in detail below:

$$
\begin{gathered}
c_{f}=\sum_{t \in T} \sum_{a \in A} \sum_{h \in H} e_{a, t}^{f, h} \cdot \xi_{a, t}^{h} \\
g_{f}=\sum_{t \in T} e_{a r r_{f}, t}^{f, h_{0}} \cdot g_{t}^{f} \\
d_{f}=\left(1-\sum_{t \in T} e_{a r r_{f}, t}^{f, h_{0}}\right) \cdot D_{f}
\end{gathered}
$$

The value of decision variable $e_{a, t}^{f, h}$ is 1 when the flight $f$ enters airway $a$ at height level $h$ at time $t$. Each airway $a \in A$ has uncertain fuel consumption $\xi_{a, t}^{h}$ for all flights.

In the formulations described above, the objective function (1a) seeks to minimize the total route cost expectation (i.e., costs for fuel consumption, arrival delay and cancellation) in the worst case. As compared to the robust ATFM model only based on the support set of the uncertain parameters, the DR-ATFM model leverages all the probability distributions $\boldsymbol{P}$ which are satisfied with certain characteristic information. As a consequence, the proposed approach is distributionally robust and can be less conservative than the contemporary robust ATFM approaches. In formulation (1), constraints (1b) describe that any flight could take off or be cancelled. But any took-off flight should land at its arrival airway. Constraints (1c) ensure that only one flight could enter one airway at the same time. Constraints (1d) denote that if one flight enters one airway inbound to a waypoint, it will enter one subsequent airway outbound from that waypoint, i.e., the flow over network is balanced. Constraints (1e) demonstrate that at each waypoint, each flight could enter at most one subsequent airway. Constraints (1f) are time connectivity constraints which means that each flight's traversing time of airway has a minimum value. Constraints (1g) ensure that the height difference between two subsequent airways within the flight routes is less than the fixed value. Constraints (1h) illustrate that the height levels of departure and arrival airport are assumed to 0 .

\section{B. Abstract Formulation}

For notation brevity, formulation (1) is given in a compact form as follows:

$$
\begin{gathered}
\min _{x}\left[\boldsymbol{a}^{T} \boldsymbol{x}+\sup _{P \in D} E_{P}\left[\xi^{T} \boldsymbol{x}\right]\right] \\
\text { s.t. } \boldsymbol{A} \boldsymbol{x} \leq \boldsymbol{b} \\
\boldsymbol{B} \boldsymbol{x}=\boldsymbol{c}
\end{gathered}
$$

where $\boldsymbol{x}=\left[e_{a, t}^{f, h}\right]^{T}$ represents the decision variables, $\xi=$ $\left[\xi_{a, t}^{h}\right]^{T}$ represents the uncertain fuel consumption of airways, constraints (5b) represent constraints (1b), (1c), (1e), (1f)and (1g), constraints $(5 \mathrm{c})$ represent constraints (1d), (1h).

\section{Ambiguity Set Construction}

An ambiguity set $D$ of candidate distributions is established, from which a distribution $\boldsymbol{P}$ or random fuel consumption $\xi$ is found to maximize the expected cost $E_{P}\left[\xi^{T} \boldsymbol{x}\right]$ given the decision $\boldsymbol{x}$ and fuel consumption $\xi$. The ambiguity set is founded in which the marginal mean vector $\mu \in R_{+}^{|I|}$ and variance $\sigma^{2} \in R_{+}^{|I|}$ of random fuel consumption are used. They are calculated by limited data as follows:

$$
\begin{gathered}
\mu_{i}:=\frac{1}{|K|} \sum_{k} d_{i}^{k} \quad \forall i \in I \\
\sigma_{i}^{2}:=\frac{1}{|K|} \sum_{k}\left(d_{i}^{k}-\mu_{i}\right)^{2} \quad \forall i \in I
\end{gathered}
$$

where $\mathrm{I}$ is the index set of $\xi$ and the size of I equals to the number of decision variables, $\mathrm{K}$ is the number of limited data samples.

The marginal moment-based ambiguity set is formulated as

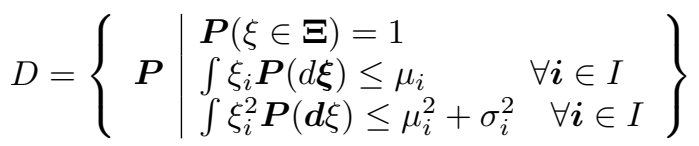

where $\boldsymbol{\Xi}$ is the support set of $\xi$ which contains various possible values of $\xi$. The three constraints of (7) ensure that (i) the integral value of $\boldsymbol{P}$ is one, (ii) true means of $\xi$ are less than calculated $\mu_{i}$ and (iii) true variances of $\xi$ are less than $\mu_{i}^{2}+\sigma_{i}^{2}$ calculated. The solution time of DR-ATFM using this set is independent from the scale of data set since the ambiguity set only use $\mu$ and $\sigma^{2}$ which are pre-calculated by (6). 


\section{Solution Methodology}

In this section, methodologies for reformulating and eventually solving the DR-ATFM model (5) using the ambiguity set (7) are elaborated.

\section{A. Reformulation of DR-ATFM}

Firstly, the worst-case expectation $\sup _{p \in D} E_{P}\left[\xi^{T} \boldsymbol{x}\right]$ in (5a) is rewrote as an optimization problem

$$
\begin{gathered}
\sup _{P \in D} E_{P}\left[\xi^{T} \boldsymbol{x}\right]=\max _{P \in D} \int \boldsymbol{\xi}^{T} \boldsymbol{x} \boldsymbol{P}(\boldsymbol{d} \xi) \\
\text { s.t. } \int \boldsymbol{\xi}_{i} \boldsymbol{P}(\boldsymbol{d} \xi) \leq \boldsymbol{\mu}_{\boldsymbol{i}} \quad \forall \boldsymbol{i} \in I \\
\int \boldsymbol{\xi}_{i}^{2} \boldsymbol{P}(\boldsymbol{d} \xi) \leq \boldsymbol{\mu}_{i}^{2}+\sigma_{i}^{2} \quad \forall \boldsymbol{i} \in I \\
\int \boldsymbol{P}(\boldsymbol{d} \xi)=1
\end{gathered}
$$

where constraints (8b) $-(8 \mathrm{~d})$ represent $P \in D$. Then above maximization problem is dualized to obtain a minimization problem that can be minimized together with the primal problem (1). Based on standard duality techniques, formulation (8) equals to the optimal objective value of the following dual formulations:

$$
\begin{gathered}
\min _{\boldsymbol{\tau} \geq 0, \boldsymbol{\alpha} \geq 0, \beta} \beta+\sum_{i}\left[\tau_{i} \mu_{i}+\alpha_{i}\left(\mu_{i}^{2}+\sigma_{i}^{2}\right)\right] \\
\text { s.t. } \quad \beta-\boldsymbol{\xi}^{\boldsymbol{T}} \boldsymbol{x}+\sum_{i}\left(\tau_{i} \xi_{i}+\alpha_{i} \xi_{i}^{2}\right) \geq 0 \quad \forall \xi \in \Xi
\end{gathered}
$$

where the dual variables $\boldsymbol{\tau} \in R^{|I|}, \boldsymbol{\alpha} \in R^{|I|}, \beta \in R$ are associated with constraints $(8 \mathrm{~b})-(8 \mathrm{~d})$, respectively. Next, the optimal problem (8) is replaced with formulation (9) in DR-ATFM model (5). And the dual formulation is merged with the primal problem to obtain the reformulation of DRATFM model (5) under the moment-based ambiguity set $D$ as follows:

$$
\begin{aligned}
\min _{\boldsymbol{x}, \boldsymbol{\tau} \geq 0, \boldsymbol{\alpha} \geq 0, \beta} \boldsymbol{a}^{T} \boldsymbol{x}+\beta+\sum_{i}\left[\tau_{i} \mu_{i}+\alpha_{i}\left(\mu_{i}^{2}+\sigma_{i}^{2}\right)\right] \\
\text { s.t. } \quad \boldsymbol{A} \boldsymbol{x} \leq \boldsymbol{b} \\
\quad \boldsymbol{B} \boldsymbol{x}=c \\
\beta \geq \boldsymbol{\xi}^{\boldsymbol{T}} \boldsymbol{x}-\sum_{i}\left(\tau_{i} \xi_{i}+\alpha_{i} \xi_{i}^{2}\right) \quad \forall \xi \in \Xi
\end{aligned}
$$

\section{B. A cutting-plane algorithm}

A cutting-plane algorithm is presented to solve the problem (10). For any $\xi$, constraint (10d) is called as a cutting plane to $\xi$. By selecting several constraints from (10d), more cutting planes are added iteratively. Then stronger relaxations are obtained until the algorithm meets the predefined conditions. A flowchart of the cutting-plane algorithm is shown in Fig. 1. And the whole algorithm is illustrated as follows:

1. Initialization of parameters sets.

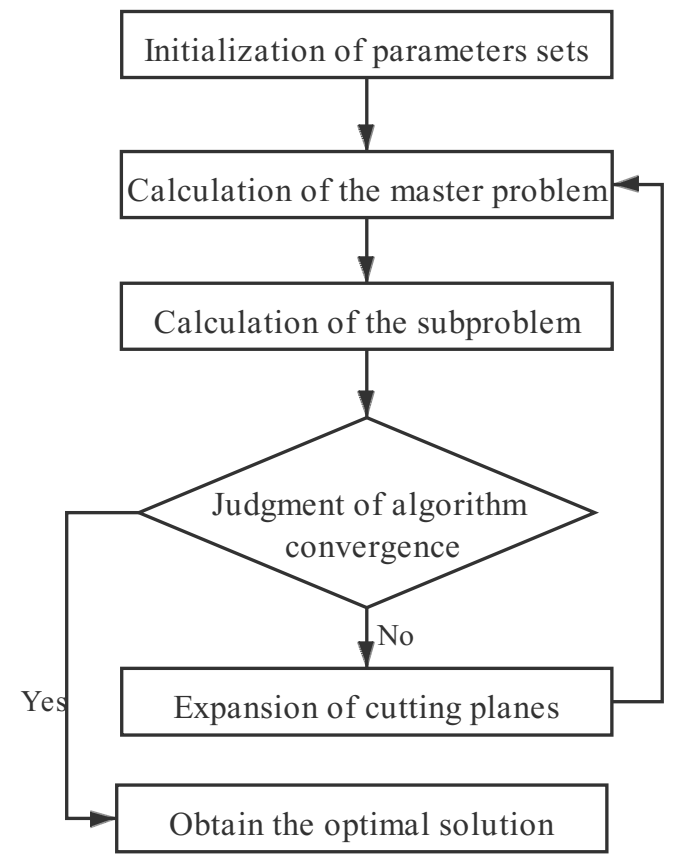

Fig. 1: Flowchart of the cutting-plane algorithm

iteration counter $N_{\text {iter }}=0$, lower bound $L B:=-\infty$, upper bound $U B:=+\infty$, set of cutting planes $C U T:=$ $\varnothing$, and select a non-negative tolerance value $\epsilon$.

2. Calculation of the master problem.

In the $m^{\text {th }}$ iteration, the master problem.

$$
\begin{gathered}
\min _{\boldsymbol{x}, \boldsymbol{\tau} \geq 0, \boldsymbol{\alpha} \geq 0, \beta} \boldsymbol{a}^{T} \boldsymbol{x}+\beta+\sum_{i}\left[\tau_{i} \mu_{i}+\alpha_{i}\left(\mu_{i}^{2}+\sigma_{i}^{2}\right)\right] \\
\text { s.t. } \quad \boldsymbol{A} \boldsymbol{x} \leq \boldsymbol{b} \\
\quad \boldsymbol{B} \boldsymbol{x}=c \\
\boldsymbol{a}^{T} \boldsymbol{x}+\beta+\sum_{i}\left[\tau_{i} \mu_{i}+\alpha_{i}\left(\mu_{i}^{2}+\sigma_{i}^{2}\right)\right] \geq 0
\end{gathered}
$$

is solved with the current set of cutting-plane cuts in $C U T$ as additional constraints. And the constraint (11d) enforces the cost lower bound. The optimal solutions $\left(\boldsymbol{x}^{m}, \boldsymbol{\tau}^{m}, \boldsymbol{\alpha}^{m}, \beta^{m}\right)$ and the optimal objective function value $V_{\text {master }}$ are recorded. Then set $L B:=V_{\text {master }}$.

3. Calculation of the subproblem.

The subproblem

$$
\max _{\boldsymbol{\xi}}\left\{\boldsymbol{\xi}^{\boldsymbol{T}} \boldsymbol{x}^{m}-\sum_{i}\left(\tau_{i}^{m} \xi_{i}+\alpha_{i}^{m} \xi_{i}^{2}\right)\right\}
$$

is solved. The optimal solution $\boldsymbol{\xi}^{m}$ and the optimal objective function value $V_{s u b}$ are recorded. Then set $U B:=L B-\beta^{m}+V_{s u b}$. It is worth noting that except for the upper and lower boundary constraints for random variables, there is no other constraint, i.e., fuel consumption can take any value between the maximum and minimum value.

4. Judgment of algorithm convergence. 
If Condition 1: $\left|\frac{U B-L B}{L B}\right| \leq \epsilon$ or Condition 2: $\beta^{m} \geq$ $V_{s u b}$, then return and output $\boldsymbol{x}^{m}$ as an optimal solution; otherwise, go to the next step.

5. Expansion of cutting planes.

Add a cutting-plane cut $\beta \geq\left(\xi^{m}\right)^{\boldsymbol{T}} \boldsymbol{x}-$ $\sum_{i}\left(\tau_{i} \xi_{i}^{m}+\alpha_{i}\left(\xi_{i}^{m}\right)^{2}\right)$ into set $C \overline{U T}$, then go to step 2 .

\section{NUMERICAL EXPERIMENTS}

In this section, case studies are performed based on smallsized instances to test the proposed DR-ATFM approach. In the solving process, the samples are solved in python language with CPLEX 12.6 on a laptop with an Intel I7-3630 QM processor, $2.40 \mathrm{GHz}, 8 \mathrm{~GB}$ RAM with a platform of Windows 10 .

\section{A. Network Configuration}

The small-sized experiments are based on 2-airport network which is composed of two airports, thirteen directed airways and six waypoints. The layout of the network is shown in Fig. 2 and the characteristics of the model are shown in Tables I-II. Noted height levels are set equal to 1, i.e., the height levels influence are not considered here in order to reduce computational complexity.

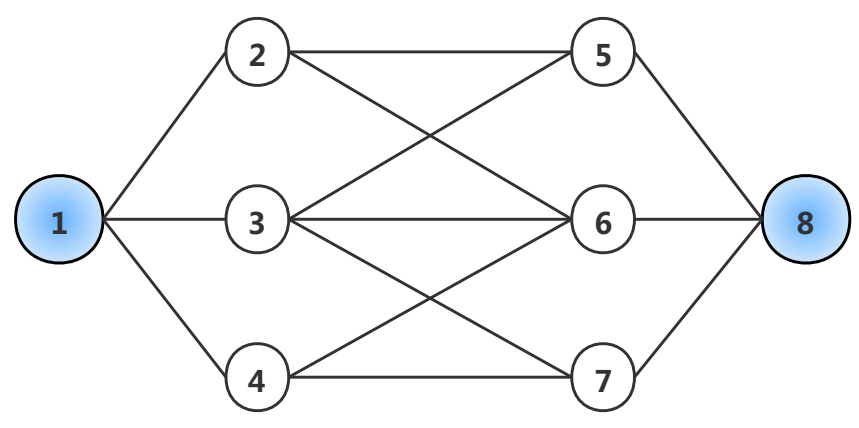

Fig. 2: 2-airport network

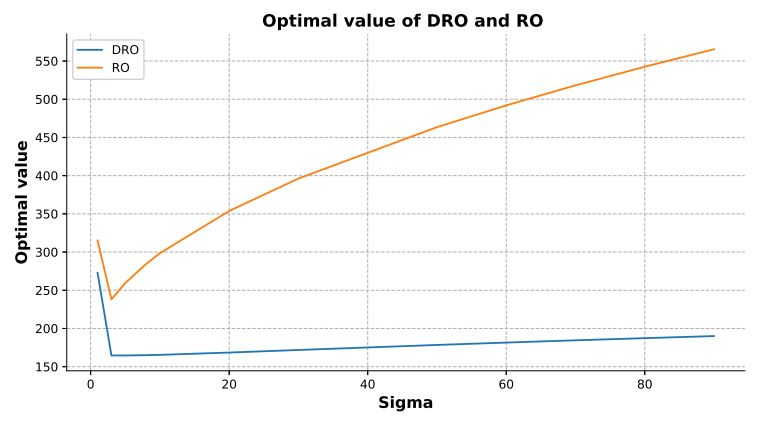

Fig. 3: The optimal value of two methods
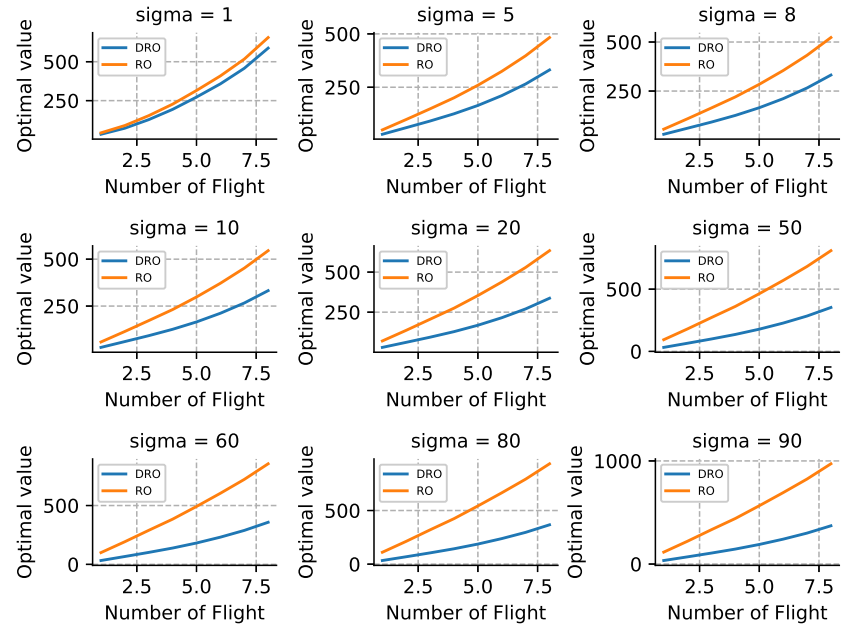

Fig. 4: The comparison of two methods under various flights and variance

TABLE I: The mean fuel consumption of airways

\begin{tabular}{|c|c|}
\hline Directed airway & The mean fuel consumption $\mu_{i}$ \\
\hline$(1,2)$ & 40 \\
$(1,3)$ & 10 \\
$(1,4)$ & 30 \\
$(2,5)$ & 50 \\
$(2,6)$ & 40 \\
$(3,5)$ & 10 \\
$(3,6)$ & 30 \\
$(3,7)$ & 20 \\
$(4,6)$ & 20 \\
$(4,7)$ & 50 \\
$(5,8)$ & 10 \\
$(6,8)$ & 20 \\
$(7,8)$ & 30 \\
\hline
\end{tabular}

\section{B. Results and Discussions}

First, the performance of DR-ATFM is studied under various fuel consumption variances which range from 1 to 50 . In particular, an out-of-sample simulation is conducted to test the optimal solutions of the DR-ATFM and the robust ATFM methods. It's suggested to note that the DR-ATFM model degenerates to robust ATFM model when the constraints (8b), (8c) of ambiguity set are ignored. In this simulation, it is assumed that there are five flights which have the same plan in the network. And the uncertain fuel consumption follows a normal distribution with independent means and variance for each flight. 300 scenarios are randomly generated. As shown in Fig. 5, the DR-ATFM problem converges when the iteration

TABLE II: The parameter of model

\begin{tabular}{|c|c|}
\hline Item & The parameter \\
\hline Flight Cancellation & $10000 \forall f$ \\
\hline Delay Cost & cost $_{\text {delay }}=\left(t-t_{\text {arrive }}\right)^{2}$ \\
\hline Time period & {$[1,90]$} \\
\hline Height & 1 \\
\hline
\end{tabular}



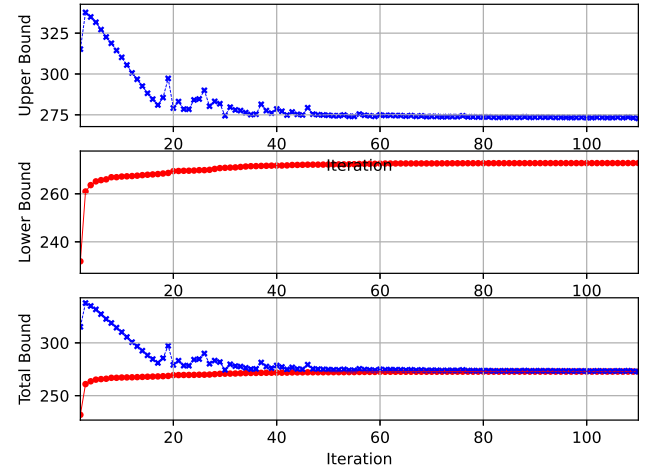

(a) $\sigma=1$

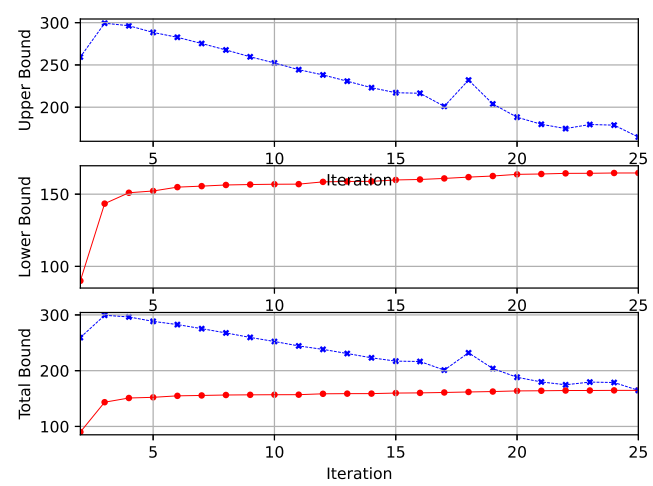

(c) $\sigma=5$
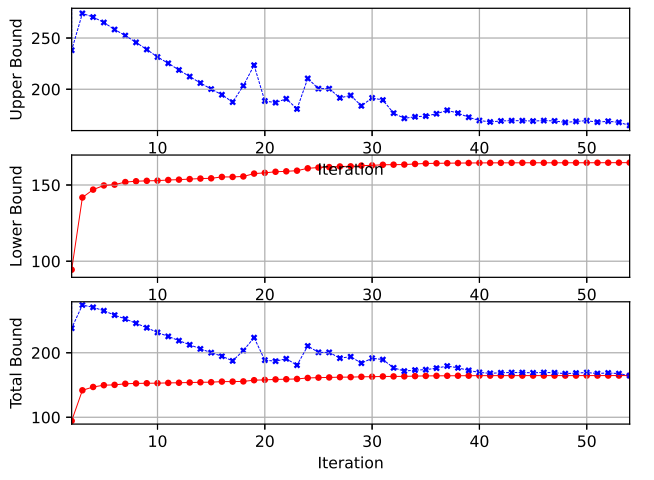

(b) $\sigma=3$

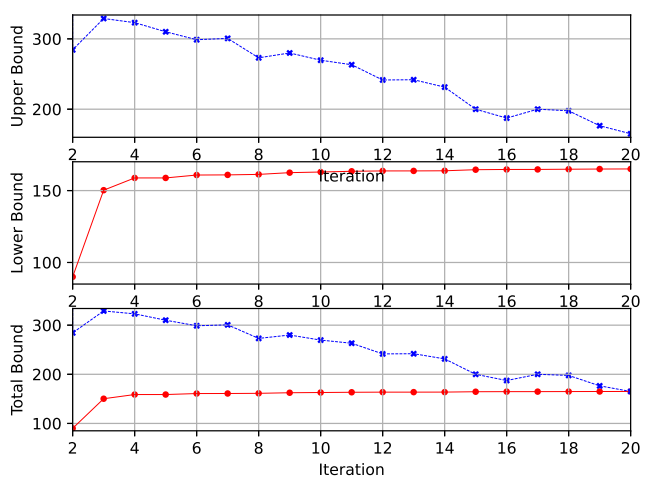

(d) $\sigma=8$

Fig. 5: An optimization of 5 flights in the network while $\sigma$ varies from 1 to 8 .

increases gradually. It could be observed that with increased iteration, the upper bound gradually decreases, and the lower bound gradually rises until they are equal, i.e., the optimal solution is obtained. And it could also be noted that the optimal value varies with the increase of variance. In Table III, the iteration number (Iter), the optimal value for DR-ATFM model (DROP) and robust model (ROOP) are reported. Particularly, Fig. 3 shows different optimal values of two methods. From Table III, it is revealed that with the increase of the variance of fuel consumption, the optimal value of DR-ATFM and robust model present growing trend. But it is clear that the growth rate of DR-ATFM is much slower than that of robust.

Second, variance is fixed to 10 and the performance of DR-ATFM under various number of flights is studied. The performance of DR-ATFM is illustrated in Table IV. From this table, it is observed that for all number of flights, the results of DR-ATFM are smaller than the ones of robust model. Then, we change variance from 1 to 80 under various number of flights. The result is shown in Fig. 4. By comparing the results of DR-ATFM model and robust model, the results of two methods are almost the same when the variance is small(e.g., $\sigma=1$ ), i.e., the models are both approximately equal to the determination model. With the increase of the variance, the gap between two optimal results become large. It is shown that DR-ATFM is less than the robust ATFM model according

TABLE III: Computational results for the 2-airport network under various means of fuel consumption

\begin{tabular}{|c|c|c|c|}
\hline The variance $\sigma$ & Iter & DROP & ROOP \\
\hline 1 & 110 & 272.8021 & 315.2092 \\
3 & 54 & 164.6595 & 238.1084 \\
5 & 25 & 164.6802 & 259.3825 \\
8 & 20 & 165.0549 & 284.3855 \\
10 & 21 & 165.4776 & 298.4771 \\
20 & 19 & 168.4779 & 353.7651 \\
30 & 19 & 171.8495 & 396.1891 \\
40 & 20 & 175.1910 & 431.9541 \\
50 & 20 & 178.4361 & 463.4638 \\
60 & 20 & 181.5425 & 491.9507 \\
70 & 21 & 184.5038 & 518.1471 \\
80 & 19 & 187.3338 & 542.5301 \\
90 & 21 & 190.0481 & 565.4312 \\
\hline
\end{tabular}

to the optimal value. In summary, the proposed DR-ATFM is less conservative than robust ATFM with the increase of number of flights. 
TABLE IV: Computational results for the 2-airport network under various number of flights

\begin{tabular}{|c|c|c|c|}
\hline The number of flights & Iter & DROP & ROOP \\
\hline 1 & 5 & 29.7082 & 58.5810 \\
2 & 8 & 60.8379 & 114.6768 \\
3 & 14 & 92.1768 & 172.5615 \\
4 & 17 & 126.1983 & 231.3833 \\
5 & 21 & 165.4776 & 298.4771 \\
6 & 23 & 211.7128 & 371.5336 \\
7 & 27 & 266.3160 & 452.1233 \\
8 & 32 & 332.2796 & 545.4255 \\
\hline
\end{tabular}

\section{CONCLUSION}

In this paper, considering the fuel consumption uncertainty of flights, a moment-based distributionally robust mixed integer programming model for the ATFM problem is proposed. The proposed DR-ATFM makes decision by minimizing the total costs of fuel consumption, flight cancellation and flight delays. Meanwhile, DR-ATFM could reduce the conservativencess by utilizing the moment information of fuel consumption. Furthermore, the model is solved by a cutting planebased decomposition algorithm. Finally, the case studies based on small-sized data verifies the reduced conservativeness of the DR-ATFM approach.

The model could be further improved in many aspects. Various kinds of uncertainty sources could be considered besides fuel consumption. For example, weather information such as radar echo value also has non-negligible impact on ATFM. Also, relevant information such covariance between the fuel consumption of different airway segments could be considered. Moreover, we could also apply the cutting plane-based decomposition algorithm to solving large-sized instances. In this way, the model could be extended to adapt to various scenarios.

\section{ACKNOWLEDGMENT}

This work is supported by the Funds of the National Natural Science Foundation of China ( Grant No. 71731001, Grant No. 61822102)

\section{REFERENCES}

[1] EUROCONTROL, "Annual network operations report 2019,"'https://www.eurocontrol.int/publication/annual-networkoperations report-2019, Accessed April, 2020.

[2] United States Department of Transportation, "Bureau of transportation statistics, airline service quality performance," https://transtats.bts.gov/,Accessed October,2020.

[3] Civil Aviation Administration of China, "Bulletin on the development of the civil aviation industry in 2019," http://www.caac.gov.cn/, Accessed June 5, 2020

[4] Bertsimas D, Lulli G, Odoni A. An integer optimization approach to large-scale air traffic flow management[J]. Operations research, 2011, 59(1): 211-227.

[5] Diao X, Chen C H. A sequence model for air traffic flow management rerouting problem[J]. Transportation Research Part E: Logistics and Transportation Review, 2018, 110: 15-30.

[6] Yu K, Kang N, Cai K, et al. Analysis and Modeling of Air Traffic Trajectories Uncertainty in Chinese Airspace[C]//2020 AIAA/IEEE 39th Digital Avionics Systems Conference (DASC). IEEE, 2020: 1-7.

[7] Agustı A, Alonso-Ayuso A, Escudero L F, et al. On air traffic flow management with rerouting. Part II: Stochastic case[J]. European Journal of Operational Research, 2012, 219(1): 167-177.

[8] Marron J B. The stochastic air traffic flow management rerouting problem[D]. Massachusetts Institute of Technology, 2004.

[9] Corolli L, Lulli G, Ntaimo L, et al. A two-stage stochastic integer programming model for air traffic flow management[J]. IMA Journal of Management Mathematics, 2017, 28(1): 19-40.

[10] Chen J, Chen L, Sun D. Air traffic flow management under uncertainty using chance-constrained optimization[J]. Transportation Research Par B: Methodological, 2017, 102: 124-141.

[11] Saraf A, Ramamoorthy K, Hunter G, et al. Robust Air Traffic Flow Management: An Optimization-Based Approach[C]//12th AIAA Aviation Technology, Integration, and Operations (ATIO) Conference and 14th AIAA/ISSMO Multidisciplinary Analysis and Optimization Conference. 2012: 5470

[12] Bertsimas D, Sim M. Robust discrete optimization and network flows[J]. Mathematical programming, 2003, 98(1): 49-71.

[13] Wiesemann W, Kuhn D, Sim M. Distributionally robust convex optimization[J]. Operations Research, 2014, 62(6): 1358-1376.

[14] Nakao H, Shen S, Chen Z. Network design in scarce data environment using moment-based distributionally robust optimization[J]. Computers operations research, 2017, 88: 44-57.

[15] Zhao C, Jiang R. Distributionally robust contingency-constrained unit commitment[J]. IEEE Transactions on Power Systems, 2017, 33(1): 94102.

[16] Zhang Y, Shen S, Mathieu J L. Distributionally robust chanceconstrained optimal power flow with uncertain renewables and uncertain reserves provided by loads[J]. IEEE Transactions on Power Systems, 2016, 32(2): 1378-1388. 